\title{
Overshunting-Related Cervical Myelopathy Causing Progressive Gait Disturbance: A Case Report
}

\author{
Eva Vister ${ }^{a}$ Sebastiaan Hammer ${ }^{b}$ Rudolf W.M. Keunen ${ }^{a}$ \\ Astrid L. Rijssenbeek ${ }^{c} \quad$ Niels A. van der Gaag ${ }^{c}$ \\ aDepartments of Neurology, Haga Teaching Hospital, The Hague, The Netherlands; \\ ${ }^{b}$ Departments of Neuroradiology, Haga Teaching Hospital, The Hague, The Netherlands; \\ 'Departments of Neurosurgery, Haga Teaching Hospital, The Hague, The Netherlands
}

\section{Keywords}

Ventriculoperitoneal shunt · Gait disturbance $\cdot$ Myelopathy $\cdot$ Venous plexus · Intracranial hypotension

\begin{abstract}
A complication of ventriculoperitoneal (VP) shunting is overdrainage or overshunting of cerebrospinal fluid, which can cause formation of hygroma but in rare cases also cervical myelopathy at a later stage. In this article, we describe a very late complication of VP shunting. We present a 75 -year-old man, previously given a VP shunt at the age of 46 , who developed a progressive gait disturbance and ataxia of the limbs after 27 years. MRI showed a cervical stenosis and myelopathy as a result of venous engorgement due to chronic overshunting of the VP shunt. Revision of the VP shunt resulted in complete resolution of his neurological symptoms and the cervical myelopathy. Cervical myelopathy due to chronic overshunting is a rare and potentially very late complication of a VP shunt. Our case underlines the importance of awareness of this complication while proper treatment can reverse the associated symptoms fully.
\end{abstract}

(C) 2021 The Author(s).

Published by S. Karger AG, Basel

\section{Introduction}

Ventriculoperitoneal (VP) shunting to divert cerebrospinal fluid (CSF) is a frequently performed and well-established treatment for different pathologies. However, the procedure is associated with a high complication risk consisting mainly of infection or malfunction. Another complication is overdrainage or overshunting of CSF, which can cause formation of 
hygroma but in rare cases also cervical myelopathy at a later stage. Chronic overshunting of CSF in the presence of a VP shunt was first described in 1998 by Miyazaki, and this sequel is also known as "the Miyazaki syndrome" [1,2]. Only a few cases are described in the literature, limiting knowledge of the exact pathophysiology and symptomatology [1,3-7]. We describe a patient who developed a symptomatic cervical stenosis with associated myelopathy 27 years after initial VP shunt placement.

\section{Case Report}

At the age of 46 , the patient presented with a progressive gait disorder, headache, nausea and vomiting. He was admitted to the hospital, and CT scan of the brain reported an obstructive hydrocephalus, caused by a dense ovoid lesion near the foramen of Monro, presumably a colloid cyst. At that time, a VP shunt procedure was performed in May 1990 (type Sigma Cordes) to reduce intracranial pressure. Postoperative CT scan showed a slit ventricle system and a residual colloid cyst (shown in Fig. 1). A few months later, the complete system had to be removed due to an infection with meningitis and subphrenic abscess formation. Follow-up CT of the brain a few weeks later showed a progressive ventricular dilatation that ultimately resulted in clinical deterioration for which a revised VP shunt procedure was performed (Unishunt with a valve pressure of 10-15 $\mathrm{cm} \mathrm{H}_{2} \mathrm{O}$ ). After recovery, the patient was readmitted again complaining of a progressive headache. A CT scan showed a left-sided large subacute subdural hematoma, opposite to the right frontal VP shunt, probably associated with an elevated international normalized ratio due to warfarin use. After normalization of the prothrombin time, he underwent an emergency decompressive craniectomy of the left frontal bone after which he recovered fully. The patient was routinely followed up until 2004 without any symptoms and normal-sized ventricles on CT. In 2007, the patient suffered from a transient episode of vertigo. MR imaging demonstrated small bilateral hygromas that could be caused by a slight overdrainage of the VP shunt.

Fig. 1. Transversal section of the brain. A dense ovoid lesion is seen in the third ventricle, near the foramen of Monro, most likely a residual colloid cyst (arrow).

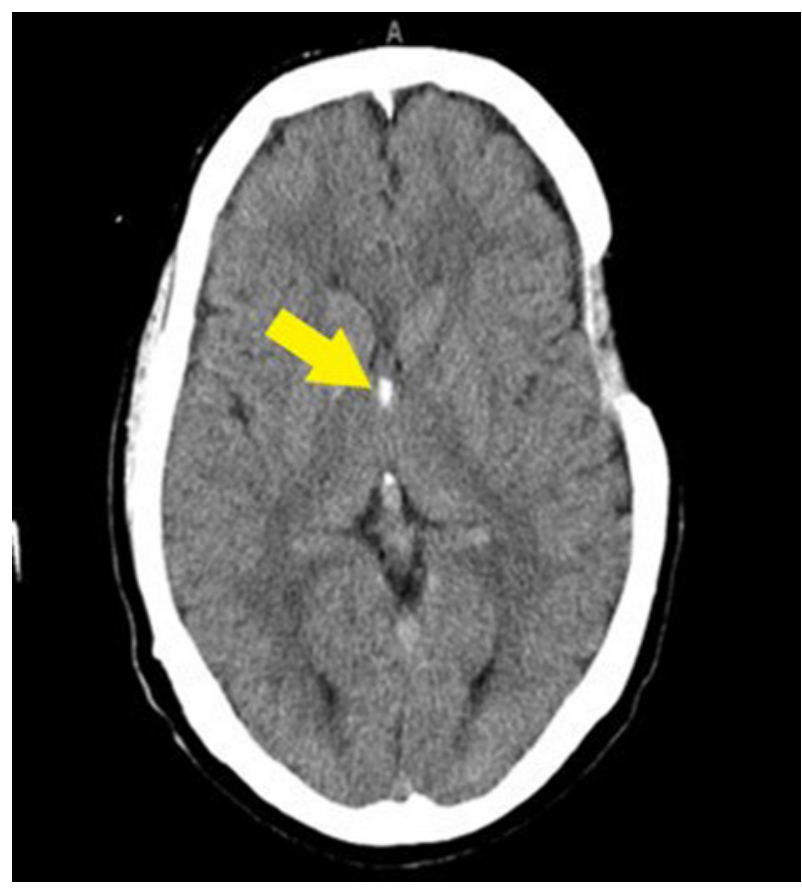


Since the patient clinically recovered without any intervention, a prolonged expectative policy to these MRI findings was conducted.

Twenty-seven years after the initial shunt surgery, the patient complained of progressive gait disturbance, increasing stiffness, and worsening dexterity of both upper limbs. Neurological examination revealed normal strength, but ataxia on both arms and legs, left more than right. Proprioception of the upper limbs was impaired, and a spastic gait was observed. An MRI of the brain (shown in Fig. 2) showed slit ventricles and bilateral hygromas equal to the MRI of 2007, suggesting VP drain overshunting. Due to the progressive gait disturbance and ataxia of the limbs, an additional MRI of the cervical spine was performed demonstrating an extensive dilatation of the suboccipital and prevertebral venous plexus with an associated very narrow central cervical canal and signs of myelopathy at the site of the craniocervical junction (shown in Fig. 3a, b). Under the assumption that overshunting led to this cascade of clinical and radiological symptoms, we initially only performed a ligation of the ventricular part of the drain to test this hypothesis and to observe any improvements. Also, the possibility that the patient had possibly redeveloped in all these years a patent CSF absorption, thus now was suffering from an overcapacity in the presence of a VP shunt, could not be ruled out. Although CT scan showed a clear decrease of the bilateral hygromas and some enlargement of the ventricles, after a few days, he worsened clinically. The option of resection of the colloid cyst at this time, which had been the initial sole reason for shunting to treat the obstruction hydrocephalus, seemed a less likely solution. Since the patient had gone through a shunt infection, hemorrhage, and long duration of shunting - all factors that can cause an acquired disturbance in CSF reabsorption - a reabsorption disturbance could not be ruled out as a factor causing the reoccurrence of hydrocephalus after ligation of the shunt. This led to a complete revision of the VP shunt (Miethke, gravity assisted valve 10/40). After the revision, the patient improved rapidly and became completely symptom free without any gait difficulties 3 months later. A follow-up MRI of the cervical spine showed (shown in Fig. 4a, b) a further increase in ventricle size to normal proportion and a fully reversed venous engorgement. Remarkably, there was even an improvement of the earlier cervical myelopathy.

Fig. 2. Coronal turbo inversion recovery magnitude MR image of the brain illustrating slit ventricles (long arrow), bilateral hygromas (short arrows), and subtle signs of myelopathy (arrow head).

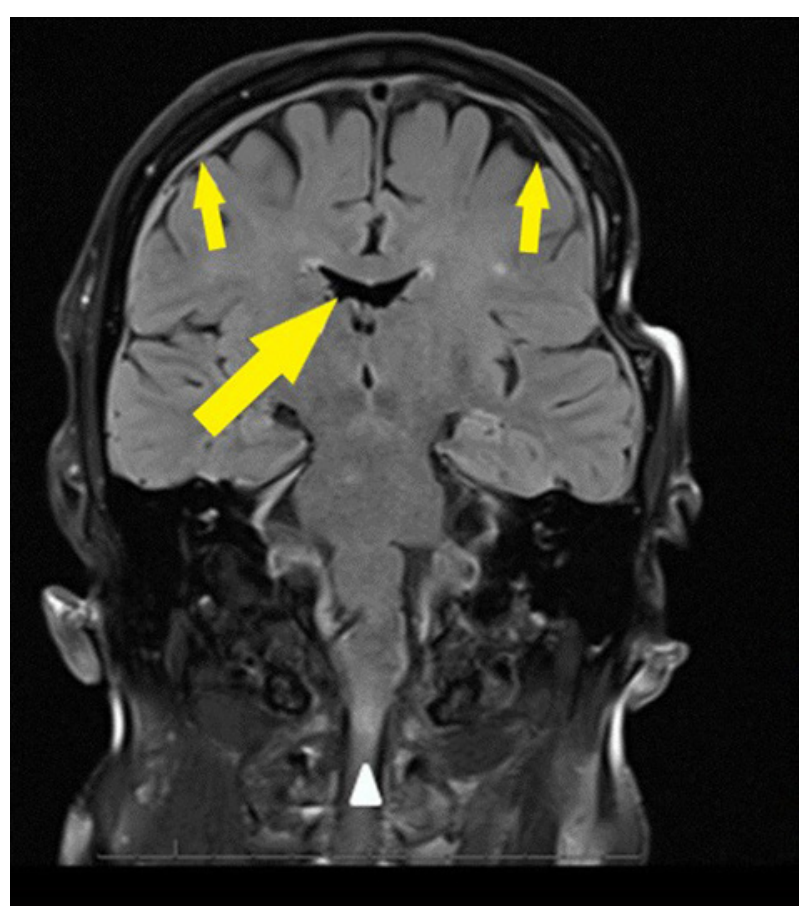




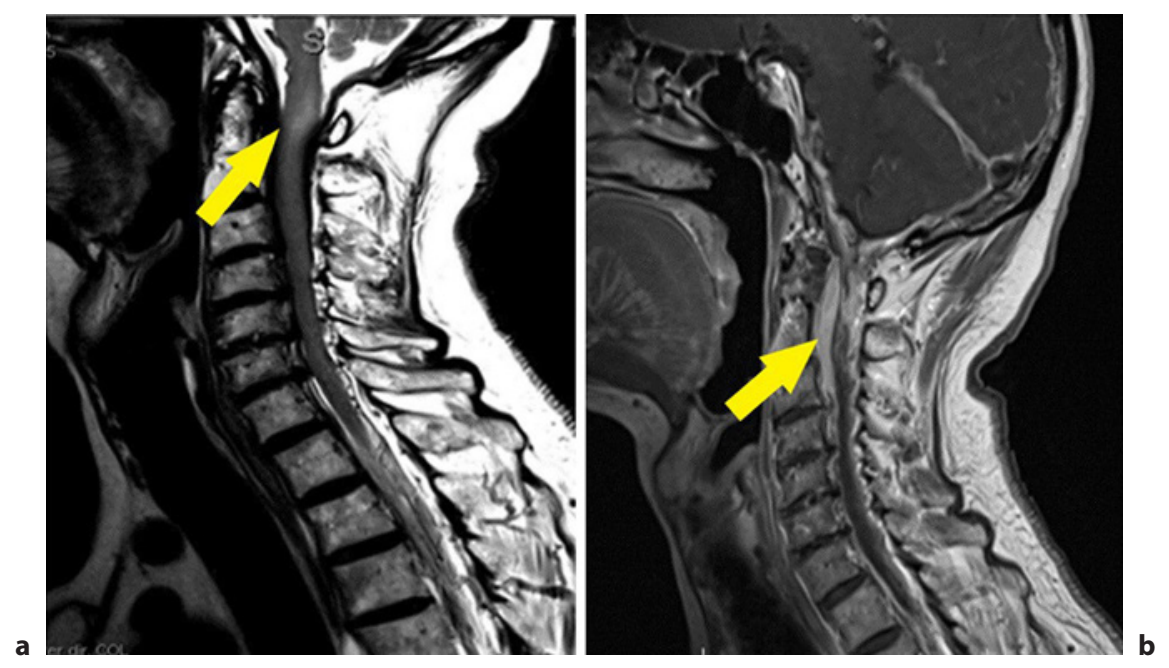

Fig. 3. a Sagittal T2 turbo spin echo scan of the cervical spine showing an area of increased signal intensity due to spinal cord compression at the $\mathrm{C} 0$-C1 level (arrow). b Extensive dilatation of the suboccipital venous plexus shown on contrast-enhanced sagittal T1 (arrow).
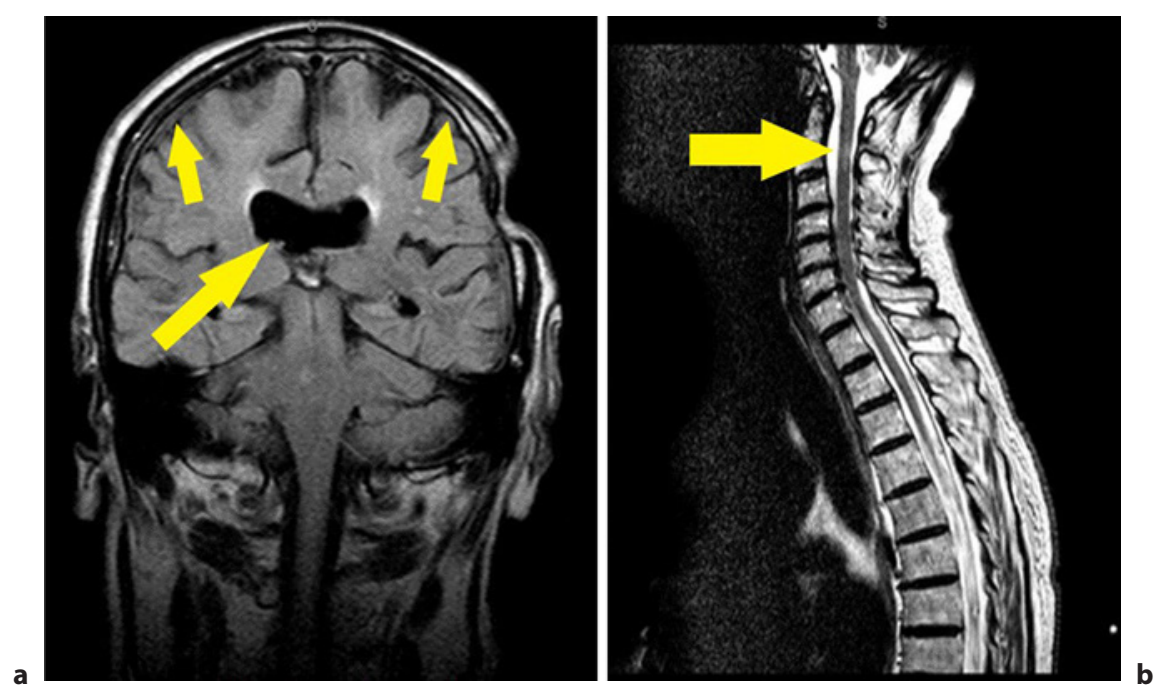

Fig. 4. a Repeated MR scan of the brain and cervical spine after VP shunt revision showed an increase in ventricle size (large arrow) and resolution of bilateral hygromas (short arrows). Further, a fully reversed venous engorgement and an improvement of the cervical myelopathy is shown in b (arrow head). Subaxially, a slight degenerative stenosis remains.

\section{Discussion}

The purpose of VP shunting in hydrocephalus is to lower intracranial pressure. Overdrainage of a shunt system is often a late complication and occurs in $10-12 \%$ of patients with ventricular shunts. Overdrainage might result in postural headaches, lethargy, nausea, and emesis but can also be entirely asymptomatic. The risk of occurrence of overdrainage due to VP shunting likely varies between individuals, depending on factors such as age, body weight, body position, and activity [8]. Subdural fluid depositions or hygroma is one of the more common symptoms of chronic overdrainage by VP shunting, mostly occurring within 12 to 24 months after shunt placement. Low intracranial pressure in VP shunting is more likely associated 
with development of a subdural hematoma. Adding an antisiphon device or placing a flowcontrol valve to increase the valve resistance of the shunt can possibly prevent overdrainage especially in the postural position. The original antisiphon valve was reported in 1973 by Portnoy et al. [9]. In 13 patients with a shunt, they demonstrated that this device was effective to prevent abnormal low intraventricular pressure.

Arnautovic et al. [10] studied the anatomical structures of the suboccipital region topographically in a cadaver study, demonstrating that the suboccipital venous plexus is located in the deep muscular layers of the neck and continues inferiorly into the deep cervical vein. This plexus is connected with the venous compartment that surrounds the vertebral artery and the transverse sigmoid sinus up through the mastoid and into occipital veins. Thus, there is a communication between upper-cervical veins and intracranial structures. Furthermore, when CSF volume decreases - due to VP shunting overdrainage - a reciprocal increase in the intracranial blood volume occurs to maintain intracranial pressure within physiological range as a mechanism of compensation. This subsequently causes swelling of the meninges and dural venous engorgement that might lead to a severe hyperproliferation of the suboccipital plexus. Chronic swelling of the meninges can induce a fibrocollagenous proliferation and contributes to dural thickening [11]. These cascades eventually can lead to compression of the spinal cord and induce a myelopathy. Depending on the body position, supine or upward position, the venous drainage of the brain follows different tracks. In the upward position, major outflow of cerebral venous drainage is seen from the internal and external posterior vertebral venous systems. The outflow through the anterior internal jugular veins seems negligible in this upward position, whereas in the supine position, the major venous outflow is through the internal jugular veins [12]. MR imaging is performed only with the patient in the supine position and thus major venous drainage in the internal jugular veins is shown. Our patient showed some degenerative cervical spinal canal stenosis with bony hook formation, so this may have aggravated the spinal cord compression. As far as we know from the literature, the very late occurrence of cervical compression and onset of symptoms, -27 years after placing the VP shunt - has not been described before. Most of the cases described in the literature showed neurological symptoms within the first 10 years after the last shunt placement. Recognition of (late) symptoms and MRI findings is crucial to establish the diagnosis.

Cervical myelopathy is generally caused by compression on the cervical spinal cord in the context of degenerative disorders or trauma. The symptoms of the patient vary in accordance with the location as well as the severity of the spinal cord compression. Early symptoms can be numbness, tingling of the hands or clumsiness, and disturbance of fine motor skills. In a later stage, loss of strength and atrophy or bladder dysfunction may be present. Clinical findings may include positive L'hermitte's sign, ataxia of arms and/or legs, exaggerated tendon reflexes, and the presence of pathological reflexes (e.g., Hoffman's sign and Babinski). High signal intensity on T2-weighted imaging intramedullary on MR imaging confirms spinal cord myelopathy. Prognosis of a diagnosed and untreated cervical myelopathy is not well characterized. As far as we know, there is no consensus regarding the timing for surgical treatment unless there is an acute deterioration, but in the context of cervical stenosis, the neurological dysfunction is likely to become irreversible without surgical intervention. Due to revision of the VP shunt, our patient fully recovered from his neurological deficit, and remarkably also the radiological signs of his myelopathy improved.

\section{Conclusion}

Cervical myelopathy due to chronic overshunting is a rare and potentially very late complication of a VP shunt. Besides regular brain imaging to monitor shunt dysfunction symptomatology, this should prompt for a low threshold to cervical spine MRI to establish this complication 
and diagnosis. The present case underlines the importance of awareness of this complication while proper treatment can reverse the associated symptoms fully after becoming symptomatic.

\section{Statement of Ethics}

Written informed consent was obtained from the patient for publication, including publication of any accompanying images.

\section{Conflict of Interest Statement}

The authors declare that they have no conflicts of interest.

\section{Funding Sources}

The authors did not receive any funding.

\section{Author Contributions}

E.V. and R.W.M.K. contributed to this work by obtaining the clinical information. E.V. drafted the manuscript. N.A.G. supervised and helped to draft the manuscript. He also performed a ligation of the ventricular part of the drain, before revision of the shunt. A.L.R. revised the VP shunt. S.H. contributed to the case by reporting the imaging of the case. All authors have read and approved the manuscript.

\section{References}

1 Caruso R, Wierzbicki V, Marrocco L, Pesce A, Piccione E. A poorly known cerebrospinal fluid shunt complication: Miyazaki syndrome. World Neurosurg. 2015;84:834-8.

2 Miyazaki T, Chiba A, Nishina H, Uesaka Y, Nakase H, Kanazawa I. Upper cervical myelopathy associated with low CSF pressure: a complication of ventriculoperitoneal shunt. Neurology. 1998;50:1864-6.

3 Ho JM, Law HY, Yuen SC, Yam KY. Overshunting-associated myelopathy: report of 2 cases. Neurosurg Focus. 2016;41:E16.

4 Martinez-lage JF, Alarcón F, Alfaro R, Ruíz-Espejo A, López-Guerrero AL, Hernández-Abenza J. Cervical extramedullary mass lesion due to chronic CSF overshunting: case report and literature review. Childs Nerv Syst. 2009;25:895-8.

5 Ulrich NH, Maier M, Bernays R, Krayenbuhl N, Kollias S. Cervical myelopathy due to chronic overshunting in a pediatric patient: case report and review of the literature. Turk Neurosurg. 2013;23:410-4.

6 Amano E, Ozaki K, Egawa S, Suzuki M, Hirai T, Ishibashi S, et al . Dynamic spinal compression revealed by computed tomography myelography in overshunting-associated myelopathy: a case report. Medicine. 2018;97:e0082.

7 Várallyay P, Nagy Z, Szucs A, Czigléczki G, Markia B, Nagy G, et al . Miyazaki syndrome: cervical myelo/radiculopathy caused by overshunting. A systematic review. Clin Neurol Neurosurg. 2019;186:105531.

8 Pudenz RH, Foltz EL. Hydrocephalus: overdrainage by ventricular shunts. A review and recommendations. Surg Neurol. 1991;35:200-12.

9 Portnoy HD, Schulte RR, Fox JL, Croissant PD, Tripp L. Anti-siphon and reversible occlusion valves for shunting in hydrocephalus and preventing post-shunt subdural hematomas. J Neurosurg. 1973;38:729-38.

10 Arnautovic KI, Al-Mefty O, Pait TG, Krisht AF, Husain MM. The suboccipital cavernous sinus. J Neurosurg. 1997; 86:252-62.

11 Mokri B, Parisi JE, Scheithauer BW, Piepgras BW, Miller GM. Meningeal biopsy in intracranial hypotension: meningeal enhancement on MRI. Neurology. 1995;45:1801-7.

12 San Mullán Ruíz D, Gailloud P, Rüfenacht DA, Delavelle J, Henry F, Fasel JH. The craniocervical venous system in relation to cerebral venous drainage. AJNR Am J Neuroradiol. 2002;23(9):1500-8. 